\title{
Surgical Management of Paediatric Neglected Humeral Supracondylar Fracture: A Series of 3 Cases in 3 Years
}

Kow Ren $\mathrm{Yi}^{1}$, Low Chooi Leng ${ }^{2}$, Nur Azuatul Akmal Kamaludin ${ }^{3}$, Aziah Abdul Aziz ${ }^{3}$, Kamariah Nor Mohd Daud ${ }^{3}$

'Department of Orthopaedic, Traumatology and Rehabilitation, International Islamic University Malaysia

2Department of Radiology, Hospital Tengku Ampuan Afzan, Kuantan Pahang

${ }^{3}$ Department of Orthopaedic Surgery, Hospital Tengku Ampuan Afzan, Kuantan Pahang.

Introduction: Supracondylar fracture of the humerus is common among paediatric population. In our country, it is not uncommon for patients to present late to the hospital after an injury. Devnani reported a case series of 28 children who sustained supracondylar humerus fracture and presented late (mean 5.6 days) to the hospital. We report our experience in managing 3 patients who presented late (range 15 days to 8 months) with neglected supracondylar humerus fracture in 3 years. Case report: Case 1: A right hand-dominant 5 -year-old boy sustained a fall 8 months prior to presentation to the hospital. He was treated with traditional methods immediately after the injury. He presented with malunion of left humeral supracondylar fracture with varus deformity and limited left elbow range of movements. An open reduction (lateral approach) and corrective osteotomy were performed and 2 lateral $\mathrm{K}$-wires were inserted. Case 2: A 10-year-old boy with right hand dominance sustained a closed left supracondylar humerus fracture Gartland 3 after a fall. His parents initially refused any surgical intervention. However, they finally agreed for surgery on day 15 post-trauma. An open reduction (posterior approach) and callus removal were done and 2 crossed $\mathrm{K}$-wires were inserted. Case 3: A right hand-dominant 5year-old girl had history of fall 1 month prior to presentation to the hospital. She was treated with traditional methods initially. She presented with malunion of the left supracondylar humerus fracture with limited range of motion of her left elbow. An open reduction (posterior approach) and corrective osteotomy were performed and 2 crossed K-wires were inserted. Discusssion: Two patients underwent open reduction via the posterior approach and 1 patient via the lateral approach. Only one patient required callus removal while 2 patients required osteoclasis. All patients recovered without complication and there was improved range of movement of the elbows. Conclusion: Surgical intervention is the treatment of choice in managing neglected supracondylar humerus fracture. 